\title{
Ocular microvascular alteration in Sjögren syndrome
}

\author{
Qi-Chen Yang ${ }^{1,2 \#}$, Fan Yao ${ }^{1 \#}$, Qiu-Yu Li ${ }^{1 *}$, Min-Jie Chen ${ }^{1}$, Li-Juan Zhang ${ }^{1}$, Hui-Ye Shu ${ }^{1}$, Rong-Bin Liang ${ }^{1}$, \\ Yi-Cong Pan $^{1}$, Qian-Min Ge ${ }^{1}$, Yi Shao ${ }^{1}$
}

${ }^{1}$ Department of Ophthalmology, The First Affiliated Hospital of Nanchang University, Jiangxi Province Ocular Disease Clinical Research Center, Nanchang, China; ${ }^{2}$ Department of Ophthalmology, West China Hospital, Sichuan University, Chengdu, China

Contributions: Conception and design: Y Shao; (II) Administrative support: Y Shao; (III) Provision of study materials or patients: QC Yang, QY Li, MJ Chen, LJ Zhang, HY Shu; (IV) Collection and assembly of data: F Yao, RB Liang, YC Pan, QM Ge; (V) Data analysis and interpretation: QC Yang; (VI) Manuscript writing: All authors; (VII) Final approval of manuscript: All authors.

\#These authors contributed equally to this work.

Correspondence to: Yi Shao. Department of Ophthalmology, The First Affiliated Hospital of Nanchang University, No 17, YongWaiZheng Street, DongHu District, Nanchang 330006, China. Email: freebee99@163.com.

Background: Sjögren syndrome $(\mathrm{SjS})$ is a systemic disease affecting exocrine, including ocular lacrimal, glands. It is uncertain whether ocular microvascular alterations are associated with this disease. In this study, we evaluated retinal and conjunctival microvascular changes in $\mathrm{SjS}$ patients using optical coherence tomography angiography (OCTA).

Methods: Twelve SjS patients (24 eyes) and 12 normal controls (24 eyes) were recruited to this study. Three-dimensional conjunctival and retinal OCTA images of each eye were captured and microvascular density was calculated. Each image was analyzed by retinal area based on the early treatment of diabetic retinopathy study method (R, S, L, and I) hemisphere segmentation method (SR, SL, IL, and IR); and central wheel division method (C1-C6). Correlation analyses were used to look for associations between retinal and conjunctival microvascular densities.

Results: Superficial and deep retinal layer microvascular density was decreased in SjS patients compared with normal controls $(\mathrm{P}<0.05)$. This significant difference was found in both superficial and deep layers in $\mathrm{S}$, L, SL, IL and C1-C3 regions, and additionally in the I and SR regions in the superficial layer. Conversely, in the conjunctiva microvascular density was higher in $\mathrm{SjS}$ patients than in controls. In $\mathrm{SjS}$ patients, a significant negative correlation was found between conjunctival and both superficial $(\mathrm{r}=-0.641 ; \mathrm{P}=0.025)$ and deep ( $\mathrm{r}=-0.958 ; \mathrm{P}<0.0001)$ microvascular densities.

Conclusions: The changed microvascular densities measured in deep and superficial retinal layers and in the conjunctiva demonstrate that OCTA is a promising method in differentiating the eyes from those with $\mathrm{SjS}$.

Keywords: Sjögren syndrome $(\mathrm{SjS})$; optical coherence tomography angiography; retinal microvascular density

Submitted Mar 02, 2021. Accepted for publication Aug 24, 2021.

doi: 10.21037/qims-21-234

View this article at: https://dx.doi.org/10.21037/qims-21-234

\footnotetext{
^ ORCID: 0000-0002-0540-5838.
} 


\section{Introduction}

Sjögren syndrome $(\mathrm{SjS})$ is a chronic systemic autoimmune disease causing inflammation of the exocrine glands (1). Patients often experience symptoms related to dryness of the mucosal surfaces, primarily in the eye and mouth. The incidence of $\mathrm{SjS}$ has been reported as 6.92 per 100,000 person-years (2), occurring about 10 times more commonly in females than males (3). SjS may have severe outcomes, such as cell lymphoma, cryoglobulinaemic vasculitis, and the SjS patients were at higher risk of death (4). Previous studies have shown retinal morphological change in SjS patients, with significantly decreased thickness of the inferior and temporal peripapillary retinal nerve fiber layer (5) and the posterior pole, as well as reduced sensitivity on perimetry (6).

The autoantibodies, especially anti-Ro/SSA antibodies, are used for early diagnosis of $\mathrm{SjS}$, but screening is important for prevention and timely treatment. The EULAR Sjögren Syndrome Patient Reported Index includes only one item related to the eye (7) illustrating a limitation of such tests which place insufficient emphasis on the dryness of the mouth and eyes, the classic symptoms of $\mathrm{SjS}$. Ocular symptoms and signs include a gritty sensation, itchiness, red appearance, and possibly dilatation of the bulbar conjunctival vessels. Studies have shown that $\mathrm{SjS}$ may lead to corneal melt, uveitis, scleritis, retinal vasculitis, and optic neuritis (8-10). In addition, one study showed that SjS patients with vision-threatening ocular findings were much more likely to have life-threatening systemic complications (11). Ocular signs and symptoms may therefore be important targets for $\mathrm{SjS}$ screening.

Optical coherence tomography angiography (OCTA) is a new imaging modality that is rapidly being adopted by eye care professionals. The principle of OCTA is to perform a continuous B-scan at one location to obtain signals reflected from blood flow and adjacent tissues. The resulting image can be divided into separate layers to accurately depict a series of vascular and structural abnormalities in the retina, choroid, and optic nerve (12). Recently, OCTA has been used in diagnosing and monitoring of eye disease, and our previous study showed that OCTA may indicate retinal microvascular pathology and visual acuity anomaly in active thyroid-associated ophthalmology (13). In an animal model of acute respiratory distress syndrome, increased retinal nerve fiber layer thickness and reactive oxygen species (HIF$1 \alpha$ and VEGF-A) were associated with increased retinal vascular permeability (14). In patients at high risk of acute coronary syndromes, retinal blood flow such as reduced inner retinal vessel density may be apparent on OCTA (15). Similarly, in patients with malignant hypertension, reduced vessel density has been found in the superficial and deep retinal layers, suggesting retinal changes associated with this disease (16).

To date, no studies have investigated retinal vessel densities in patients with $\mathrm{SjS}$. The present study addressed this gap, using OCTA to assess whether ocular vessel density is altered in patients with active SjS.

\section{Methods}

\section{Research subjects}

In this case-control study, 12 patients (24 eyes) diagnosed with $\mathrm{SjS}$ were recruited at the First Affiliated Hospital of Nanchang University between June 2020 to September 2020 (4 months) and 12 normal controls ( 24 eyes). All subjects were examined by the same retinal specialist. The person who knows the diagnoses details would not participate in the OCTA examination and analyses. The normal control group included the individuals with no ocular or systemic diseases which could affect the eye circulation, like diabetic.

\section{Recruitment criteria}

Patients meeting the following criteria were eligible to enter the study: (I) female and (II) diagnosed with SjS by rheumatologist with disease duration of 1-7 years, SSA and RO-52 antibody positive.

Patients meeting any of the following criteria were not eligible: (I) Systemic autoimmune diseases, such as systemic lupus erythematosus. (II) Ocular surgery within the previous 6 months. (III) Circulatory diseases affecting the eye, such as hypertension or diabetes. (IV) Eye diseases, such as glaucoma or keratoconus. (V) Pregnant or lactating. (VI) Contraindication for pupil dilatation or intolerance to topical anesthetic or mydriatics or (VII) smoker. (VIII) Without using any eyedrops.

\section{Ethical considerations}

This study was conducted in accordance with the Declaration of Helsinki (as revised in 2013). The study was approved by the ethics committee of the First Affiliated Hospital of Nanchang University (No. 2020039). Informed 
consent was required from all subjects prior to participation.

\section{Clinical examinations}

All patients underwent clinical and ophthalmological assessments (without using anesthesia) including autoantibody testing (17), and monocular assessment of visual acuity, Schirmer test, ocular staining score (OSS), tear breakup time (tBUT), tear meniscus height (18) intraocular pressure and OCTA.

The Schirmer test was conducted with a duration of five minutes. The ocular staining score (OSS) was calculated based on fluorescein staining in each quadrant of the cornea as follows: 0 points $=$ no spot staining; 1 point $=1-3$ spot staining; 2 points $\geq 4$ spot staining; 3 points $=$ spot staining fusion or appearance of filaments. The quadrant scores were summed, giving a total score of $0-12$ points. Tear breakup time (tBUT) was assessed using fluorescein sodium and subjective observation of time to observation of tear film rupture. Tear meniscus height was measured objectively using the Keratograph $5 \mathrm{M}$ corneal topographer.

\section{OCTA}

All angiography was conducted by the same examiner using the Angio OCT Optvue RTVue XR Avanti system (Optovue, Fremont, CA). The scan speed was set to 70,000 A-scans per second, center wavelength $840 \mathrm{~nm}$, bandwidth $45 \mathrm{~nm}$, axial resolution $5 \mu \mathrm{m}$ and horizontal resolution $22 \mu \mathrm{m}$. B-scan (along the $\mathrm{x}$-axis) in a $6 \times 6-\mathrm{mm}$ scan pattern with 5 repetitions of angiography was used for imaging at 216 raster positions (along the y-axis), focusing on the fovea. The acquisition time was $3.9 \mathrm{~s}$. We captured a $1,080 \mathrm{~b}$-scan (216y position $\times 5$ position) at 270 frames per second. Each person who did the OCTA test was asked to open the eyes as large as possible and hold still for at least $10 \mathrm{~s}$. Three-dimensional $6 \times 6 \mathrm{~mm}$ en-face OCTA images were recorded from each eye, as in our previous study (13).

Vessels were segmented using the split-spectrum amplitude-decorrelation algorithm. The retinal capillary network was manually segmented into two physiologic layers: (I) Superficial retinal layer (SRL), between the vitreoretinal interface and the anterior boundary of the ganglion cell layer. (II) Deep retinal layer (DRL), between the inner boundary of the inner plexiform layer and the outer boundary of the outer plexiform layer. In each layer, the macrovascular (SMAR and DMAR respectively), microvascular (SMIR and DMIR) and total microvascular (STMI and DTMI) networks were analyzed. The vessel density was calculated as described previously (13) (Figure 1A-1H).

The macular retinal area was segmented as follows (Figure 1I-1P) (13): (I) Using the early treatment of diabetic retinopathy study (ETDRS) method the image was divided into right (R), left (L), superior (S) and inferior (I) quadrants (Figure 17,1N). (II) Using the hemisphere segmentation method, the image was divided into diagonal quadrants, superior right (SR), superior left (SL), inferior right (IR) and inferior left (IL) (Figure 1K,1O). (III) Using the central wheel division method, the central foveal area with diameter of $0.6 \mathrm{~mm}$ was disregarded and an annulus adjacent to this with diameter of $2.5 \mathrm{~mm}$ and width of $0.95 \mathrm{~mm}$ was defined. The annulus was then divided into 6 rings (C1-C6) each with a bandwidth of $0.16 \mathrm{~mm}$, for analysis (Figure $1 \mathrm{~L}, 1 \mathrm{P}$ ).

For the conjunctival OCTA images, the beam width was set to $22 \mu \mathrm{m}$, the axial resolution to $5 \mu \mathrm{m}$ and light source center to $840 \mathrm{~nm}$. The speed of scanning was to 70,000 times per second. The lens adapter was set to 2 to $4 \mathrm{~cm}$ from the corneal surface of the participant. The images were exported to ImageJ (National Institutes of Health, Bethesda, MD). About 4mm along the circumference of the edge of the limbus conjunctivae and 5 consecutive rectangles (100 pixels, $850 \mu \mathrm{m})$ were identified as scanned areas. The microvascular structure was highlighted by selected filter to generate binary images for analysis (Figure 2).

\section{Statistical analysis}

The data were analyzed using statistical software (Statistica version 7.1, StatSoft, Inc., Tulsa, OK; and MedCalc software version 10, MedCalc Software, Mariakerke, Belgium). Data are presented as mean \pm SD. The data of two eyes in one person was averaged. Comparisons between groups were performed using an independent-sample $t$-test, and $\mathrm{P}$ values less than 0.05 were considered statistically significant. A receiver operating characteristic (ROC) curve was used to compare micro-vessel density of the superficial and deep retinal layers between the two groups.

\section{Results}

\section{Baseline characteristics of the control and SjS groups}

To test whether the two groups were similar in aspects other than those related to $\mathrm{SjS}$, subjects' age and blood 

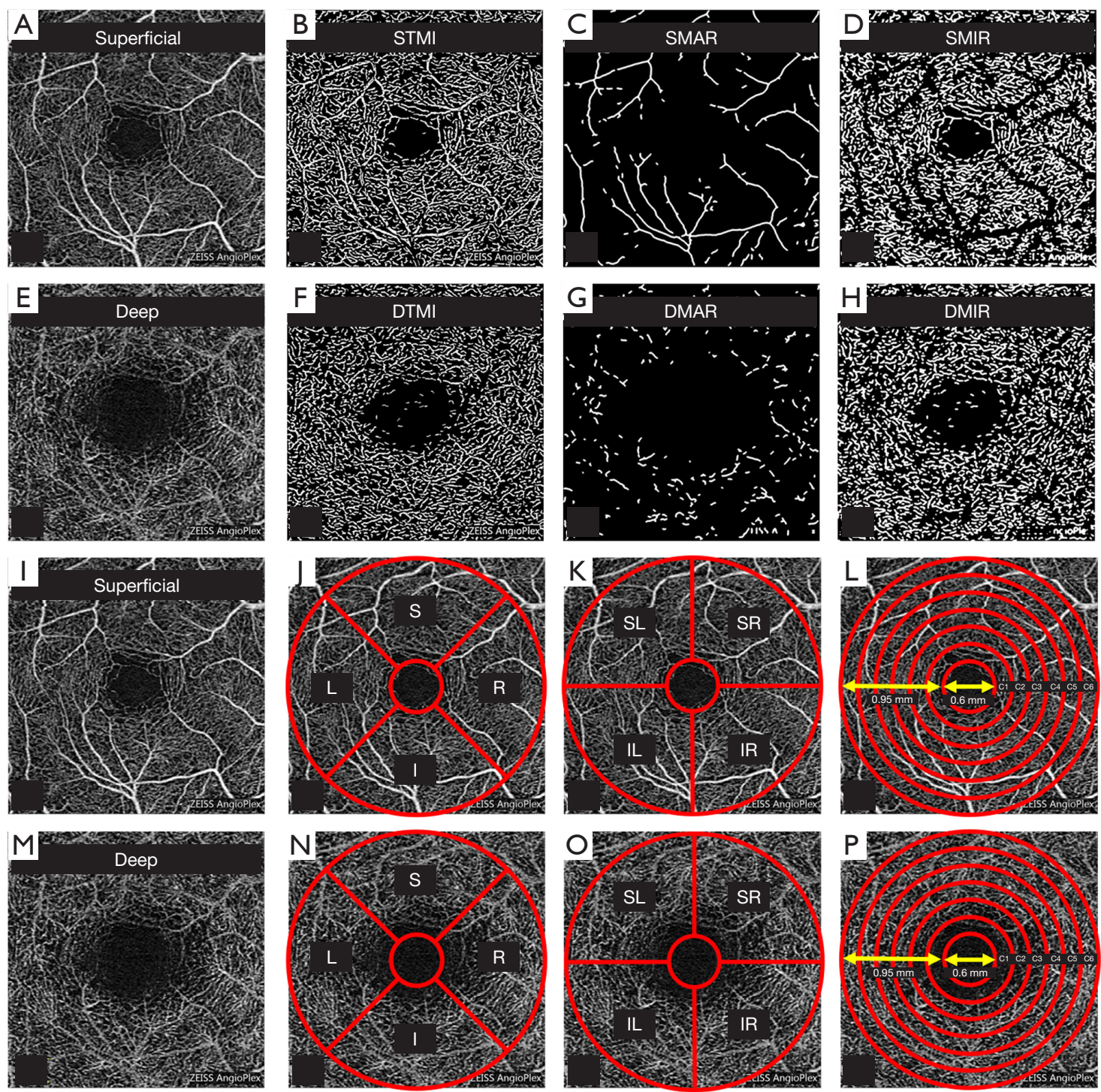

Figure 1 The optical coherence tomography angiography image of the macular region of the retina (A-H). Partition methods of the retinal microvascular (I-P). SjS, Sjögren syndrome; STMI, superficial total microvascular; SMAR, superficial macrovascular; SMIR, superficial microvascular; DTMI, deep total microvascular; DMAR, deep macrovascular; DMIR, deep microvascular.
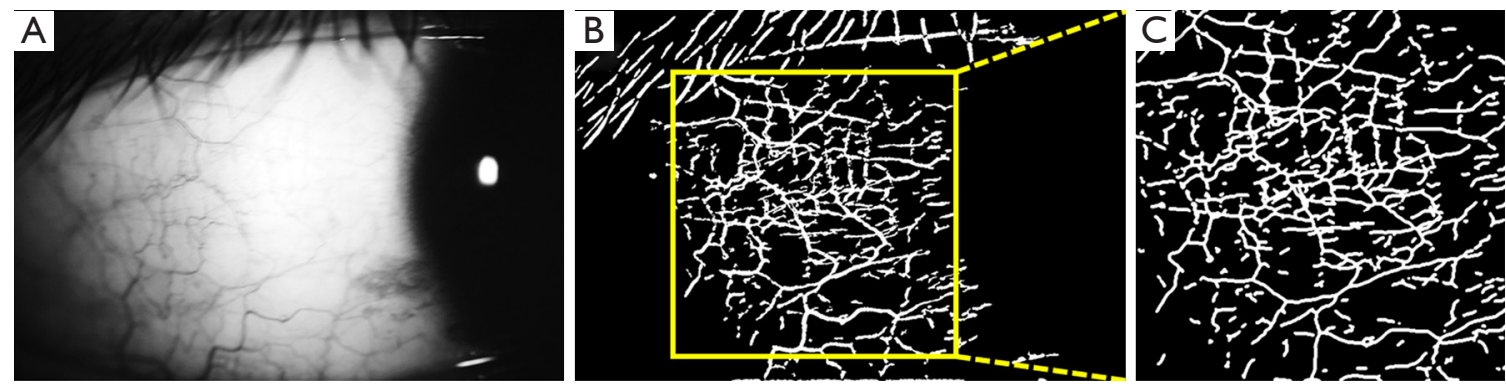

Figure 2 The optical coherence tomography angiography image of the microvascular of the conjunctiva. 
Table 1 General information of normal control group and SjS group

\begin{tabular}{lcccc}
\hline & Age (year) & Disease duration (year) & Systolic blood pressure $(\mathrm{mmHg})$ & Diastolic blood pressure $(\mathrm{mmHg})$ \\
\hline Control & $54.50 \pm 9.05$ & 0 & $123.08 \pm 4.25$ & $83.33 \pm 6.67$ \\
SjS & $55.17 \pm 9.48$ & $3.92 \pm 1.89$ & $126.92 \pm 5.48$ & $78.67 \pm 6.42$ \\
$t$ & 0.17 & - & 1.83 & 1.67 \\
$\mathrm{P}$ & 0.868 & - & 0.081 & 0.109 \\
\hline
\end{tabular}

SjS, Sjögren syndrome.

Table 2 Ocular clinical findings of normal control group and SjS group

\begin{tabular}{lcccccc}
\hline & Average visual acuity & Average IOP $(\mathrm{mmHg})$ & Average tBUT $(\mathrm{s})$ & Average OSS & Average SIT $(\mathrm{mm})$ & Average TMH $(\mathrm{mm})$ \\
\hline Control & $0.89 \pm 0.09$ & $15.13 \pm 1.31$ & $13.42 \pm 1.17$ & 0 & $12.92 \pm 0.84$ & $0.58 \pm 0.10$ \\
SjS & $0.66 \pm 0.18$ & $14.63 \pm 1.35$ & $4.67 \pm 0.75$ & $3.13 \pm 1.24$ & $3.33 \pm 1.18$ & $0.15 \pm 0.02$ \\
$t$ & 3.862 & 0.882 & 20.92 & 8.33 & 21.98 & 86.85 \\
$\mathrm{P}$ & 0.0008 & 0.388 & $<0.0001$ & $<0.0001$ & $<0.0001$ & $<0.0001$ \\
\hline
\end{tabular}

SjS, Sjögren syndrome; IOP, intraocular pressure; tBUT, tear breakup time; OSS, ocular staining score; SIT, Schirmer I test; TMH, tear meniscus height.

pressure were recorded. Disease duration was also recorded in the SjS group. No significant difference in age or blood pressure was found between groups, as shown in Table 1.

\section{Ocular clinical findings}

No significant differences in intraocular pressure was found between the two groups. In the $\mathrm{SjS}$ group, the average visual acuity was $0.66 \pm 0.18$, which was significantly lower than the normal control group $(0.89 \pm 0.09, \mathrm{P}=0.0008)$. The tBUT $(4.67 \pm 0.75 \mathrm{~s})$ in $\mathrm{SjS}$ group was significantly shorter than in the normal control group $(13.42 \pm 1.17 \mathrm{~s}, \mathrm{P}<0.0001)$. The OSS was increased in the $\mathrm{SjS}$ group, at $3.13 \pm 1.24$ and the Schirmer test showed lower tear production in the $\mathrm{SjS}$ group $(3.33 \pm 1.18 \mathrm{~mm})$ than in the normal group $(12.92 \pm 0.84 \mathrm{~mm}$, both $\mathrm{P}<0.0001)$. Tear meniscus height (TMH) also showed the same trend, with $0.15 \pm 0.02 \mathrm{~mm}$ in the $\mathrm{SjS}$ group and $0.58 \pm 0.10 \mathrm{~mm}$ in the normal group $(\mathrm{P}<0.0001)$ (Table 2).

\section{Superficial macular vascular density}

Microvascular, macrovascular ring and total microvascular densities in the superficial layer were compared between groups (Figure $3 A, 3 B)$. The STMI $(\mathrm{P}<0.0001)$ and SMIR $(\mathrm{P}<0.0001)$ densities were significantly decreased in the SjS group, but SMAR $(\mathrm{P}=0.0777)$ density was similar in the two groups (Figure $3 C)$. The $\mathrm{SR}(\mathrm{P}=0.0001), \mathrm{SL}(\mathrm{P}<0.0001)$, and IL $(\mathrm{P}<0.0001)$ regions (hemisphere segmentation method; Figure $3 D$ ) were significantly lower than that in control group. Vessel density was significantly lower in the $\mathrm{SjS}$ group than in controls in the $\mathrm{S}(\mathrm{P}=0.0003), \mathrm{I}(\mathrm{P}=0.0097)$, and $\mathrm{L}(\mathrm{P}<0.0001)$ regions (using the EDTRS method; Figure $3 E)$ and in $\mathrm{C} 1(\mathrm{P}<0.0001), \mathrm{C} 2(\mathrm{P}=0.0001)$ and $\mathrm{C} 3$ $(\mathrm{P}<0.0023)$ regions (central wheel division method; Figure $3 F)$. Between-group differences did not reach significance in other regions.

\section{Deep macular vascular density}

Microvascular, macrovascular ring and total microvascular densities in the deep layer were compared between groups (Figure 4A,4B). Similarly, DTMI $(\mathrm{P}=0.0011)$ and DMIR $(\mathrm{P}<0.0001)$ densities were significantly lower in $\mathrm{SjS}$ than in the control group, but DMAR $(\mathrm{P}=0.876)$ was similar in the two groups (Figure $4 C$ ). Blood vessel density in SL $(\mathrm{P}=0.0002)$ and IL $(\mathrm{P}<0.0001)$ regions (hemisphere segmentation method; Figure $4 D$ ) were significantly lower in $\mathrm{SjS}$ than that in control. Microvascular density in $\mathrm{S}$ $(\mathrm{P}=0.0008)$ and $\mathrm{L}(\mathrm{P}=0.0001)$ regions (EDTRS method; Figure $4 E)$, and in $\mathrm{C} 1(\mathrm{P}=0.0013), \mathrm{C} 2(\mathrm{P}<0.0001)$ and $\mathrm{C} 3$ $(\mathrm{P}=0.0364)$ regions (central wheel division; Figure $4 F)$ were significantly lower in SjS than in controls. Density in other regions was statistically similar in the two groups. 
A
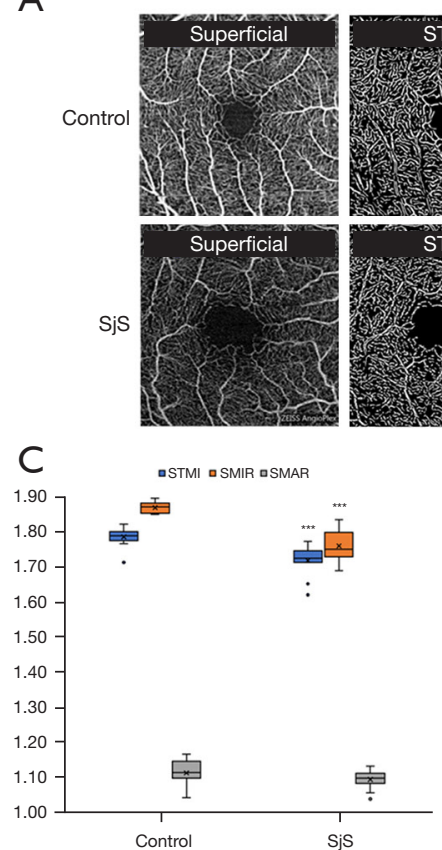
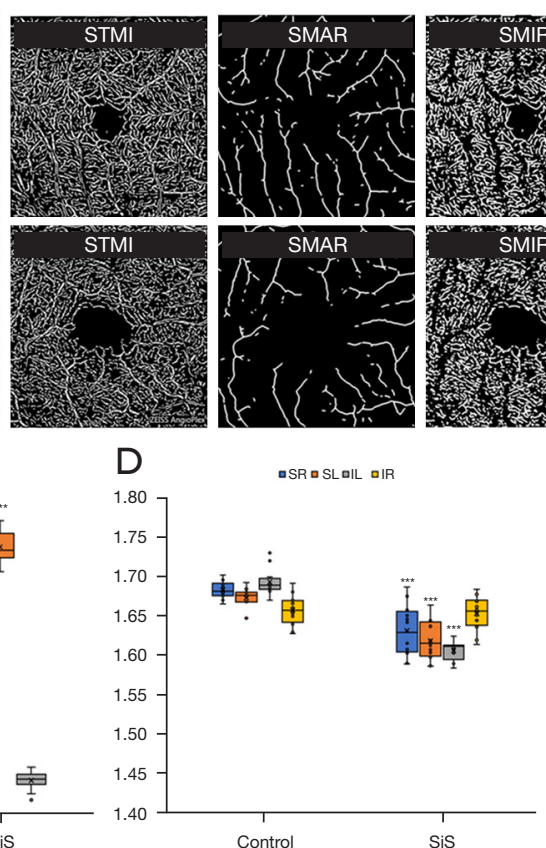
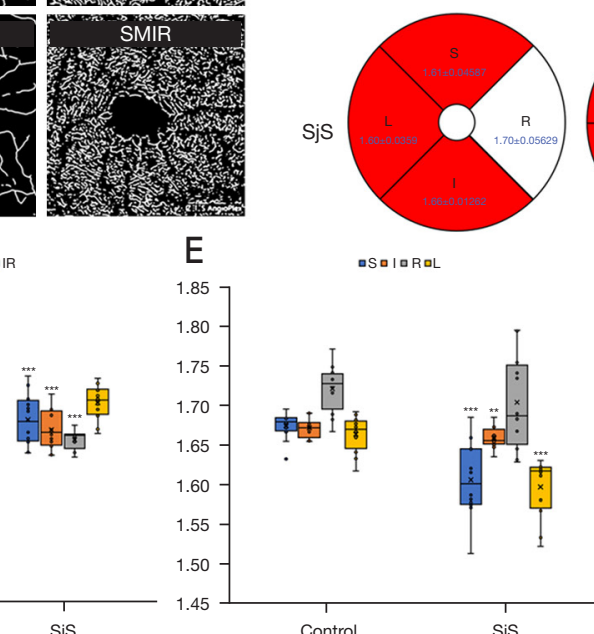

SjS

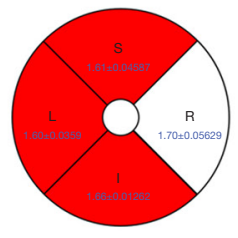

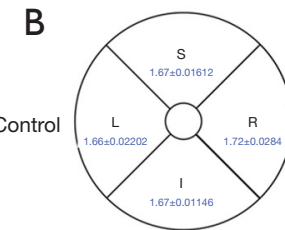
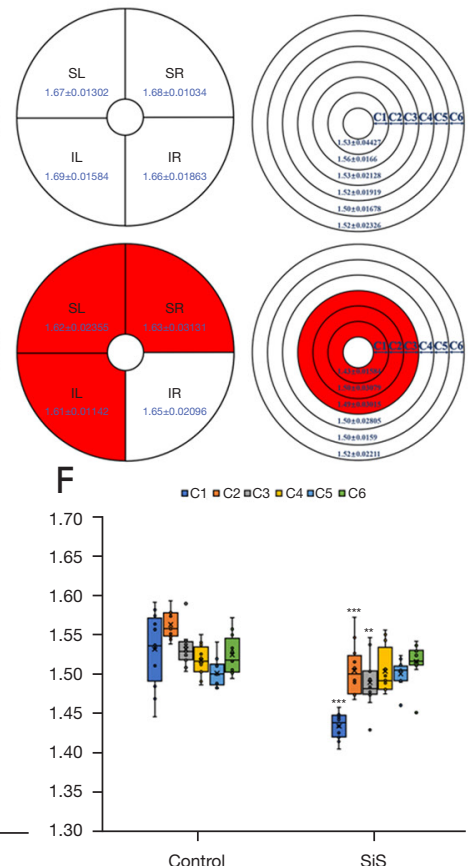

Figure 3 Comparison of retinal vessel density between $\mathrm{SjS}$ and control groups in superficial retinal layers. The optical coherence tomography angiography image of the superficial retinal layer (A). Superficial retinal microvascular in different partition methods(B). Box plot of microvascular densities in superficial retinal layer (C). Box plot of microvascular densities in hemisphere segmentation method (D). Box plot of microvascular densities in EDTRS method (E). Box plot of microvascular densities in central wheel division (F). SjS, Sjögren syndrome; STMI, superficial total microvascular; SMAR, superficial macrovascular; SMIR, superficial microvascular; R, right; L, left; S, superior; I, inferior; SR, superior right; SL, superior left; IR, inferior right; IL, inferior left. ${ }^{*} \mathrm{P}<0.05 ;{ }^{* *} \mathrm{P}<0.01 ;{ }^{* * *} \mathrm{P}<0.001$.

\section{ROC analysis of superficial and deep retinal vessel densities}

OCTA measurement of retinal vessel density showed high specificity and sensitivity in differentiation between the SjS and normal control groups (Figure 5). In the superficial retinal layer, significant differences between groups were found in the STMI, SMIR, SR, SL, IL, S, I, L, and C1-C3 regions. Among them, the areas under the ROC curves for SMIR and IL superficial retinal densities were $1.0[95 \%$ confidence interval (CI): 1], indicating high sensitivity in the diagnosis of SjS (Figure 5A). The DTMI, DMIR, SL, IL, S, $\mathrm{L}$, and $\mathrm{C} 1-\mathrm{C} 3$ regions in the deep retinal vessel layer were also significantly different between groups. The areas under the ROC curves of DMIR, IL, and C2 were 0.951 (95\% CI: 0.864-1), demonstrating high diagnostic sensitivity of deep retinal density in these regions (Figure $5 B$ ).

\section{Conjunctival vascular density}

The temporal conjunctival vessel density was measured using OCTA in the two groups and was significantly higher in $\mathrm{SjS}$ than in normal controls $(\mathrm{t}=3.138 ; \mathrm{P}=0.0048)$ (Figure $6 A)$. The ROC analysis also showed a significant difference in conjunctival vessel density between the two groups. The area under the ROC curve was 0.191 (95\% CI: 0.016-0.366) (Figure 6B).

\section{Relationship between retinal and conjunctival blood vessel densities}

We investigated the relationships between retinal and conjunctival blood vessel densities. In the superficial retinal layer of the SjS group, the correlation coefficient of STMI region and temporal conjunctival vessel density was -0.641 (Figure $7 A$ ). Similarly, in the deep retinal layer, the correlation coefficient was -0.958 (Figure $7 B$ ). In the normal control group, only the DTMI region in the superficial retinal layer showed a significant negative correlation with conjunctival vessel density, with the correlation coefficient was -0.761 (Figure 7). 
A
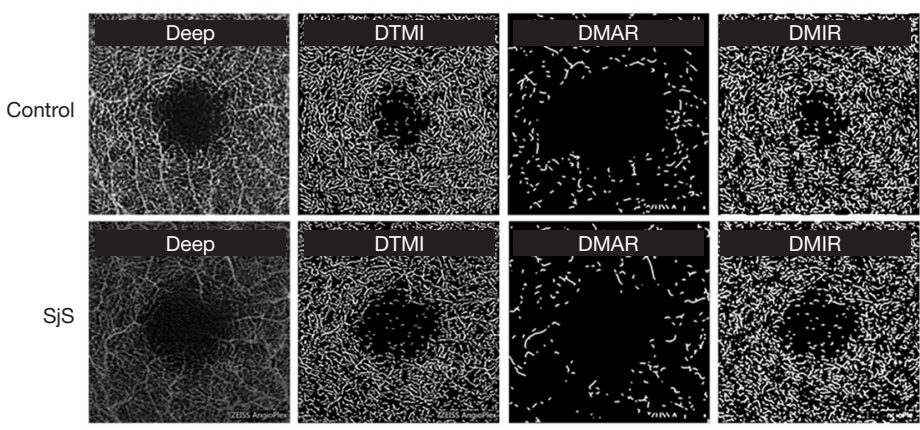
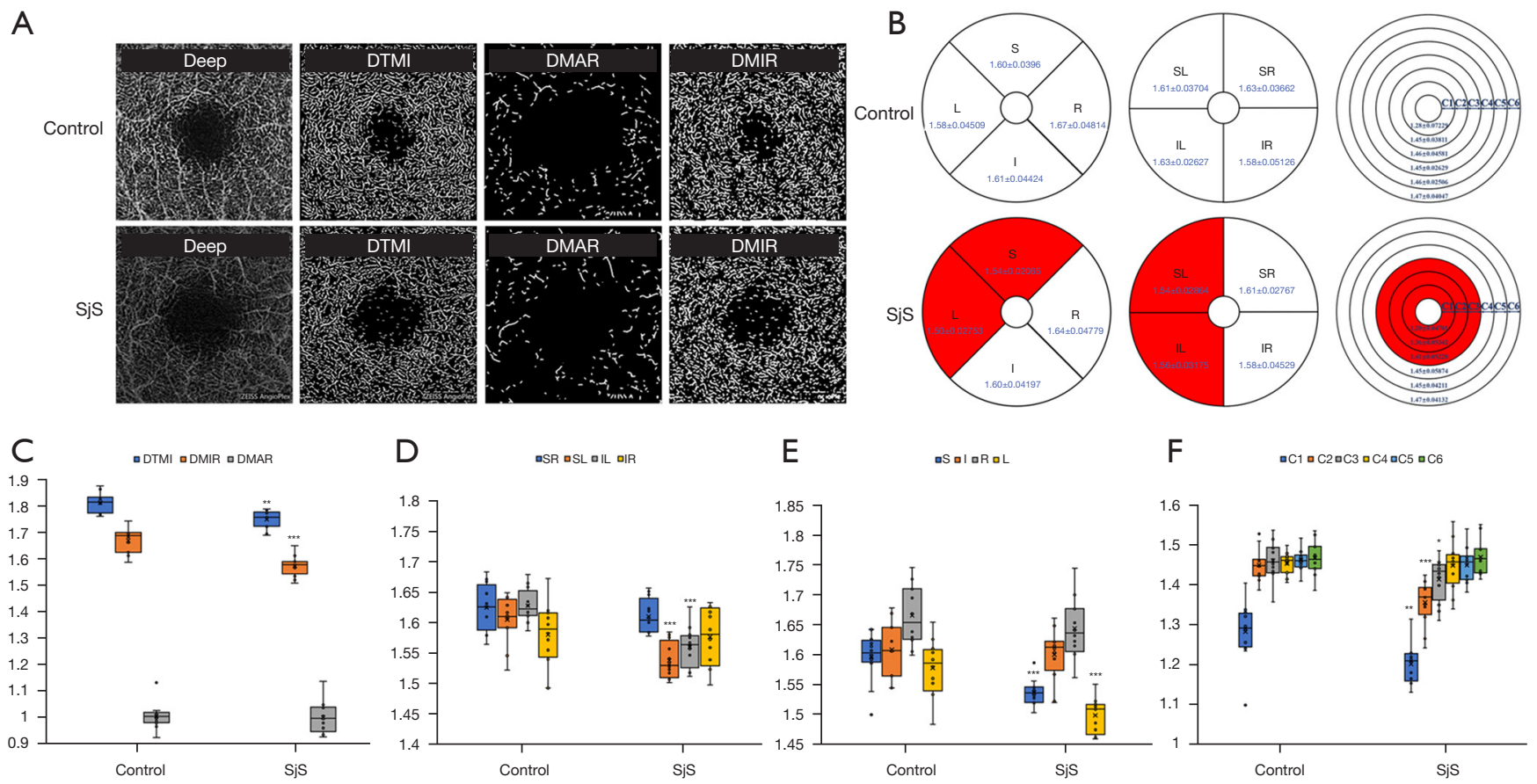

Figure 4 Comparison of retinal vessel density between SjS and control groups in deep retinal layers. The optical coherence tomography angiography image of the deep retinal layer (A). Deep retinal microvascular in different partition methods (B). Box plot of microvascular densities in deep retinal layer (C). Box plot of microvascular densities in hemisphere segmentation method (D). Box plot of microvascular densities in EDTRS method (E). Box plot of microvascular densities in central wheel division (F). SjS, Sjögren syndrome; DTMI, deep total microvascular; DMAR, deep macrovascular; DMIR, deep microvascular; R, right; L, left; S, superior; I, inferior; SR, superior right; SL, superior left; IR, inferior right; IL, inferior left. ${ }^{*} \mathrm{P}<0.05 ;{ }^{* *} \mathrm{P}<0.01 ;{ }^{* * *} \mathrm{P}<0.001$.

\section{Discussion}

This is the first published study on OCTA measurements of ocular microvascular changes in $\mathrm{SjS}$ patients. The study confirmed signs of dry eye in patients with $\mathrm{SjS}$, found decreased microvascular but not macrovascular density compared with normal controls, identified potential diagnostic markers, and the results suggested a negative association between retinal and conjunctival blood vessel density

$\mathrm{SjS}$ is a systemic autoimmune disease, which is characterized by lymphocytic infiltration and influences the secretory glands. Dry eye and mouth are common symptoms of $\mathrm{SjS}$ (1). The diagnosis of $\mathrm{SjS}$ is based on the American-European consensus group classification, which includes: (I) ocular symptoms; (II) oral symptoms; (III) ocular signs (schirmer's test and ocular dye score); (IV) histopathology; (V) salivary gland involvement; (VI) autoantibodies (antibodies to Ro/SSA and/or La/SSB antigens). The presence of any four of these six items is indicative of $\mathrm{SjS}(19)$. Studies have found that $\mathrm{SjS}$ is diagnosed in middle age. Research based in Spain found the mean age of SjS patients to be 62 years in 1997 (20) but in 2018 it was reported as 53 years (21). The present study also showed this trend, as the age of the $\mathrm{SjS}$ patients was $55.17 \pm 9.48$ years.

The eye is one of the prominent organs affected by $\mathrm{SjS}$ and dryness of the ocular surface in this condition may be observed directly. According to the Dry Eye Workshop (DEWS) II, tBUT $<10 \mathrm{~s}$, Schirmer test $<10 \mathrm{~mm} / 5 \mathrm{~min}$ and tear meniscus height $<0.15 \mathrm{~mm}$ are indicators of dry eye (18). In our study, all the SjS patients had positive findings of dry eye tests. We also found the visual acuity was significantly decreased in $\mathrm{SjS}$ patients when compared with the normal control group, while the intraocular pressure was not significantly changed. Previous studies have shown that $\mathrm{SjS}$ patients have not only ocular surface dryness but also fundus damage, such as optic neuropathy (22), ischemic choroidopathy (23) and optic neuritis (24) in addition to reduced retinal thickness and visual field sensitivity (6). 

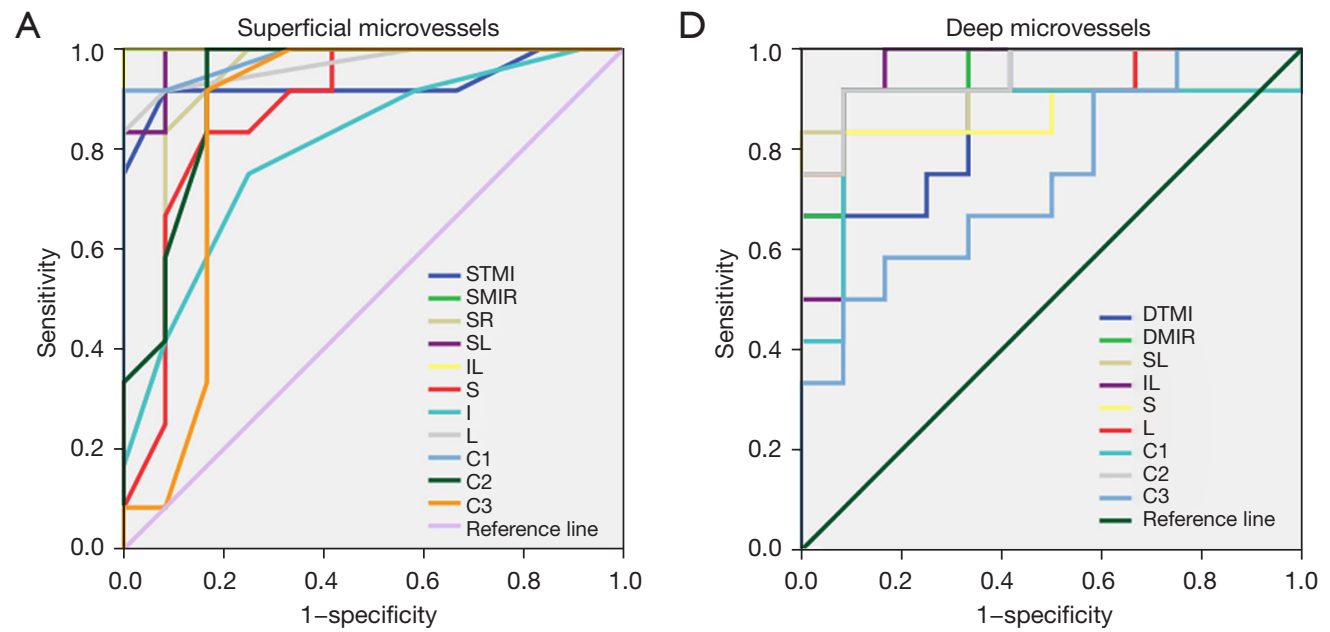

Figure 5 ROC curve analysis of different retinal partition microvascular densities in the superficial (A) and deep layers (B). STMI, superficial total microvascular; SMAR, superficial macrovascular; SMIR, superficial microvascular; DTMI, deep total microvascular; DMAR, deep macrovascular; DMIR, deep microvascular; R, right; L, left; S, superior; I, inferior; SR, superior right; SL, superior left; IR, inferior right; IL, inferior left.

A

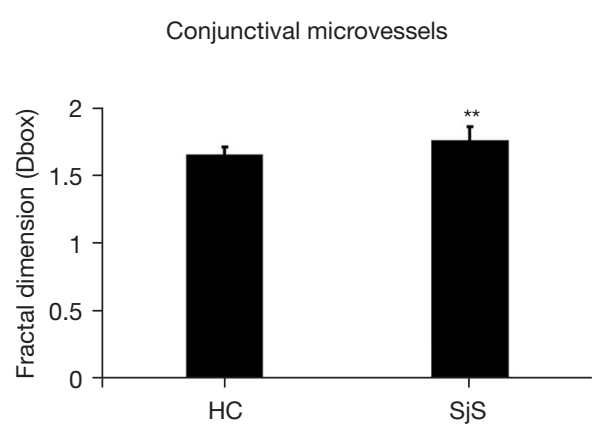

B

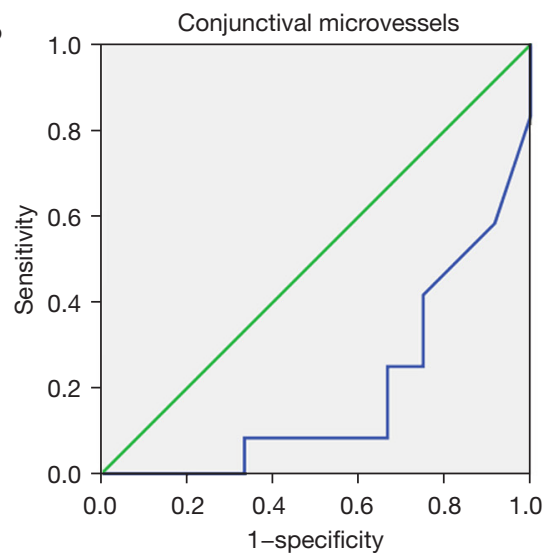

Figure 6 Conjunctival vessel density between $\mathrm{SjS}$ and control groups. Histogram analysis of $\mathrm{SjS}$ and control groups (A). ROC curve analysis between two groups (B). HC: healthy control; SjS, Sjögren syndrome. ${ }^{* *} \mathrm{P}<0.01$.

Anti-Sjögren's syndrome type B (SSB) antibody positivity was associated with peripapillary retinal nerve fiber layer thickness reduction, and anti-SSB positivity $(\mathrm{P}=0.005)$ and erythrocyte sedimentation rate (ESR) level was associated with macular ganglion cell-inner plexiform layer thickness reduction (25).

As $\mathrm{SjS}$ is a chronic disease, pathophysiological changes in blood vessels have been reported in previous studies. In a study of $25 \mathrm{SjS}$ patients, capillary density and the diameters of the apical, afferent and efferent capillaries were increased in $\mathrm{SjS}$ patients compared with control subjects (26). Another study showed that in $\mathrm{SjS}$ patients moderate avascularity was the most common finding, and that steadystate cutaneous perfusion was significantly decreased (27). Vasculitis associated with antibodies to Ro /SSA has also been reported in SjS patients (28). Angiogenesis has been closely linked to the progression of $\mathrm{SjS}$, and could represent a novel potential biomarker of SjS disease activity (29). OCTA is a non-invasive, time-efficient measurement that could provide insight on the perfusion of vascular networks 
A

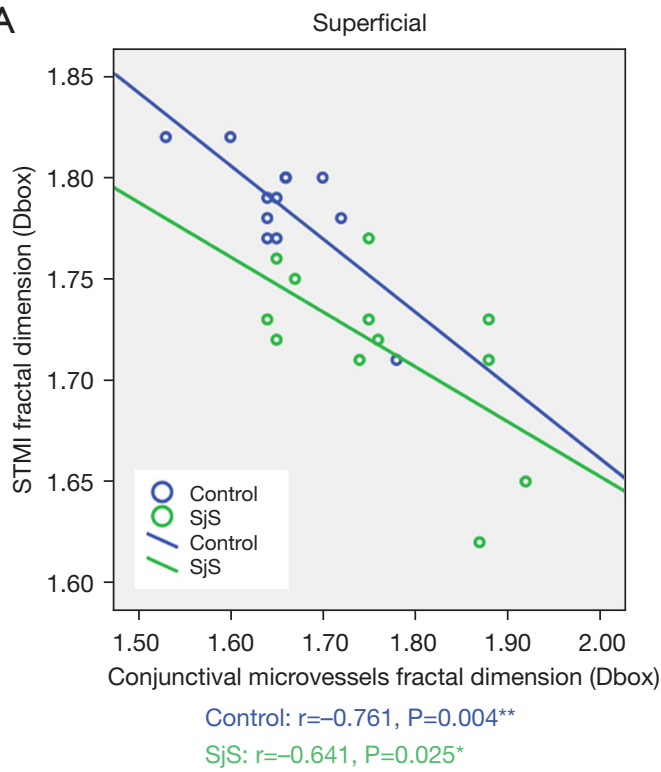

B

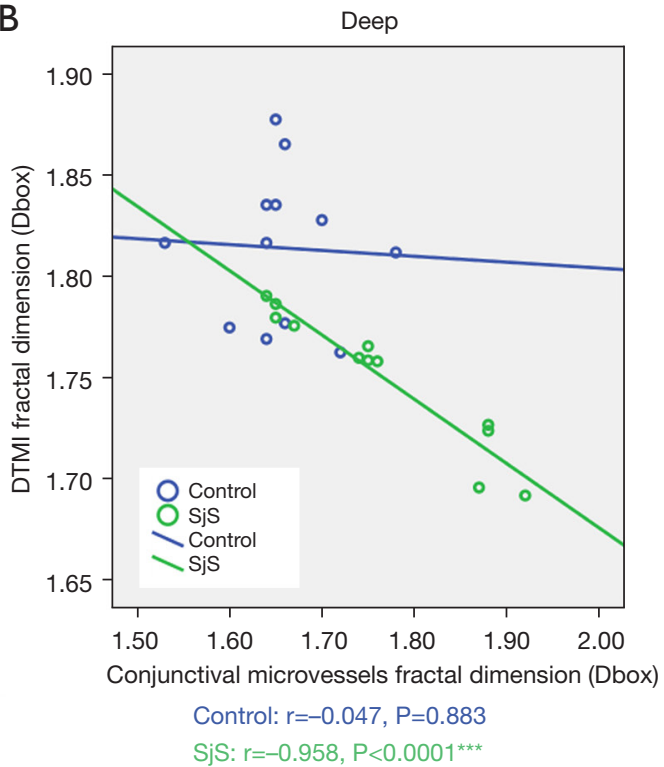

Figure 7 Correlation analysis of total microvascular in both superficial and deep retinal layers in SjS and control groups. SjS, Sjögren syndrome.

in the eye, providing an indication of microvascular health without the use of dye, and is a relatively safe examination for patients.

In our study, we found the density of conjunctival microvasculature was increased significantly in $\mathrm{SjS}$. Consistent with our findings, previous studies have shown that neovascularization in $\mathrm{SjS}$ is regulated by increases in vascular endothelial growth factor-A (VEGF-A) and its receptor vascular endothelial growth factor receptor-2 (VEGFR-2) (30) in addition to upregulation of the Neuropilin-1 (31).

The new finding in our study was the retinal microvascular changes in $\mathrm{SjS}$ patients. Our results showed that macular STMI and SMIR densities were decreased in $\mathrm{SjS}$ patients compared to normal controls, while the density of SMAR did not show the same trend. In the deep retinal layer, the densities of DTMI and DMIR were also decreased, and decreased microvascular and total microvascular density was found in the $\mathrm{C} 1, \mathrm{C} 2$ and C3 regions. A previous study of age-related macular degeneration showed that microvascular density reduction was significantly related to a decrease in inner retinal thickness (32). In unilateral retinal vein occlusion an association has been found between peripapillary vessel density retinal nerve fiber and ganglion cell-inner plexiform layer thickness (33). In another systemic disease, diabetic mellitus, retinal microvascular change and retinal structure degeneration have been found to be closely related (34) and systemic vascular changes indicate subclinical retinal ischemia with neurodegeneration (35).

In $\mathrm{SjS}$ patients, previous work has found decreased retinal thickness, especially in the retinal nerve fiber layer and macular ganglion cell-inner plexiform layer (5). The perfusion abnormality of retinal microvasculature could lead to development of inflammatory infiltrates in the larger vessels, resulting in retinal thickness decrease (36). The results showed in our study demonstrated the correlation between the retinal microvascular and conjunctival microvascular in $\mathrm{SjS}$ patients. The mechanisms were still unknown and we hypothesized that in $\mathrm{SjS}$ patients, some inflammatory factors could play as an angiogenic factors to the conjunctival microvascular whereas these factors could cause the retinal thickness and retinal microvascular decrease. The previous study showed some inflammation molecules, such as thrombospondin-1 (TSP-1) and CD31 could cause angiogenesis in the conjunctiva (37) and cornea (38). Moreover, it was also proved that the TSP had degeneration ability of retinal microvascular (39). However, further studies are needed to verify our hypothesis.

There were some limitations in our study. In the normal control group, the average visual acuity was $0.89 \pm 0.09$, and the mean age of the control group was $54.50 \pm 9.05$. The 
decreased visual acuity of the control group might due to the cataract, but it still needed to find out the reason that causes the visual acuity of the control group decrease. In this study, we only recruited women, as $\mathrm{SjS}$ was a disease with a female-to-male predominance of 9:1 and peak incidence at approximately 50 years of age (40). For further study, the male also needs to include. In this study, we did not do the multiple testing, and more data needed to be collected.

\section{Conclusions}

In the present study, we characterized the ocular microvascular changes in SjS patients using OCTA. The results showed a decrease in macular microvascular density in both superficial and deep retinal layers. While the sample size in this study was not sufficient to allow analysis by disease stage, the changed microvascular densities measured in deep and superficial retinal layers and in the conjunctiva demonstrate that OCTA is a promising method in differentiating the eyes from those with SjS.

\section{Acknowledgments}

Funding: Key Research Foundation of Jiangxi Province (20203BBG73059,20181BBG70004); Excellent Talents Development Project of Jiangxi Province (20192BCBL23020); Natural Science Foundation of Jiangxi Province (20181BAB205034); Grassroots Health Appropriate Technology "Spark Promotion Plan" Project of Jiangxi Province (20188003); Health Development Planning Commission Science Foundation of Jiangxi Province (20201032); Health Development Planning Commission Science TCM Foundation of Jiangxi Province (2018A060).

\section{Footnote}

Conflicts of Interest: All authors have completed the ICMJE uniform disclosure form (available at https://dx.doi. org/10.21037/qims-21-234). The authors have no conflicts of interest to declare.

Ethical Statement: The authors are accountable for all aspects of the work in ensuring that questions related to the accuracy or integrity of any part of the work are appropriately investigated and resolved. The study was conducted in accordance with the Declaration of Helsinki (as revised in 2013). The study was approved by the ethics committee of the First Affiliated Hospital of Nanchang
University (No. 2020039) and informed consent was taken from all the patients.

Open Access Statement: This is an Open Access article distributed in accordance with the Creative Commons Attribution-NonCommercial-NoDerivs 4.0 International License (CC BY-NC-ND 4.0), which permits the noncommercial replication and distribution of the article with the strict proviso that no changes or edits are made and the original work is properly cited (including links to both the formal publication through the relevant DOI and the license). See: https://creativecommons.org/licenses/by-nc-nd/4.0/.

\section{References}

1. Brito-Zerón P, Baldini C, Bootsma H, Bowman SJ, Jonsson R, Mariette X, Sivils K, Theander E, Tzioufas A, Ramos-Casals M. Sjögren syndrome. Nat Rev Dis Primers 2016;2:16047.

2. Qin B, Wang J, Yang Z, Yang M, Ma N, Huang F, Zhong R. Epidemiology of primary Sjögren's syndrome: a systematic review and meta-analysis. Ann Rheum Dis 2015;74:1983-9.

3. Ramos-Casals M, Brito-Zerón P, Kostov B, Sisó-Almirall A, Bosch X, Buss D, Trilla A, Stone JH, Khamashta MA, Shoenfeld Y. Google-driven search for big data in autoimmune geoepidemiology: analysis of 394,827 patients with systemic autoimmune diseases. Autoimmun Rev 2015;14:670-9.

4. Brito-Zerón P, Kostov B, Solans R, Fraile G, SuárezCuervo C, Casanovas A, Rascón FJ, Qanneta R, PérezAlvarez R, Ripoll M, Akasbi M, Pinilla B, Bosch JA, NavaMateos J, Díaz-López B, Morera-Morales ML, Gheitasi H, Retamozo S, Ramos-Casals M; SS Study Group, Autoimmune Diseases Study Group (GEAS), Spanish Society of Internal Medicine (SEMI). Systemic activity and mortality in primary Sjögren syndrome: predicting survival using the EULAR-SS Disease Activity Index (ESSDAI) in 1045 patients. Ann Rheum Dis 2016;75:348-55.

5. Yang JM, Heo H, Park SW. Relationship between retinal morphological findings and autoantibody profile in primary Sjögren's syndrome. Jpn J Ophthalmol 2014;58:359-68.

6. Conigliaro P, Triggianese P, Draghessi G, Canofari C, Aloe G, Chimenti MS, Valeri C, Nucci C, Perricone R, Cesareo M. Evidence for the Detection of Subclinical Retinal Involvement in Systemic Lupus Erythematosus and Sjögren Syndrome: A Potential Association with 
Therapies. Int Arch Allergy Immunol 2018;177:45-56.

7. Seror R, Ravaud P, Mariette X, Bootsma H, Theander E, Hansen A, et al. EULAR Sjogren's Syndrome Patient Reported Index (ESSPRI): development of a consensus patient index for primary Sjogren's syndrome. Ann Rheum Dis 2011;70:968-72.

8. Zheng W, Liu X, Hou X, Zhu Y, Zhang T, Liao L. Recurrent optic neuritis in a patient with Sjogren syndrome and neuromyelitis optica spectrum disorder: A case report. Medicine (Baltimore) 2020;99:e23029.

9. Watanabe T, Marumo Y, Ishizu A. Retinal vasculitis in primary Sjögren's syndrome. Rheumatology (Oxford) 2019;58:1244.

10. Vivino FB, Minerva P, Huang CH, Orlin SE. Corneal melt as the initial presentation of primary Sjögren's syndrome. J Rheumatol 2001;28:379-82.

11. Akpek EK, Mathews P, Hahn S, Hessen M, Kim J, GraderBeck T, Birnbaum J, Baer AN. Ocular and systemic morbidity in a longitudinal cohort of Sjögren's syndrome. Ophthalmology 2015;122:56-61.

12. Sambhav K, Grover S, Chalam KV. The application of optical coherence tomography angiography in retinal diseases. Surv Ophthalmol 2017;62:838-66.

13. Ye L, Zhou SS, Yang WL, Bao J, Jiang N, Min YL, Yuan Q, Tan G, Shen M, Shao Y. RETINAL MICROVASCULATURE ALTERATION IN ACTIVE THYROID-ASSOCIATED OPHTHALMOPATHY. Endocr Pract 2018;24:658-67.

14. Zadeh JK, Ruemmler R, Hartmann EK, Ziebart A, Ludwig M, Patzak A, Xia N, Li H, Pfeiffer N, Gericke A. Responses of retinal arterioles and ciliary arteries in pigs with acute respiratory distress syndrome (ARDS). Exp Eye Res 2019;184:152-61.

15. Arnould L, Guenancia C, Azemar A, Alan G, Pitois S, Bichat F, Zeller M, Gabrielle PH, Bron AM, CreuzotGarcher C, Cottin Y. The EYE-MI Pilot Study: A Prospective Acute Coronary Syndrome Cohort Evaluated With Retinal Optical Coherence Tomography Angiography. Invest Ophthalmol Vis Sci 2018;59:4299-306.

16. Courtie E, Veenith T, Logan A, Denniston AK, Blanch RJ. Retinal blood flow in critical illness and systemic disease: a review. Ann Intensive Care 2020;10:152.

17. Jonsson R, Theander E, Sjöström B, Brokstad K, Henriksson G. Autoantibodies present before symptom onset in primary Sjögren syndrome. JAMA 2013;310:1854-5.

18. Wolffsohn JS, Arita R, Chalmers R, Djalilian A, Dogru M, Dumbleton K, Gupta PK, Karpecki P, Lazreg S, Pult
H, Sullivan BD, Tomlinson A, Tong L, Villani E, Yoon KC, Jones L, Craig JP. TFOS DEWS II Diagnostic Methodology report. Ocul Surf 2017;15:539-74.

19. Both T, Dalm VA, van Hagen PM, van Daele PL. Reviewing primary Sjögren's syndrome: beyond the dryness - From pathophysiology to diagnosis and treatment. Int J Med Sci 2017;14:191-200.

20. Ramos M, Cervera R, García-Carrasco M, Miret C, Muñoz FJ, Espinosa G, Font J, Ingelmo M. Primary Sjögren's syndrome: clinical and immunologic study of 80 patients. Med Clin (Barc) 1997;108:652-7.

21. Brito-Zerón P, Acar-Denizli N, Ng WF, Zeher M, Rasmussen A, Mandl T, et al. How immunological profile drives clinical phenotype of primary Sjögren's syndrome at diagnosis: analysis of 10,500 patients (Sjögren Big Data Project). Clin Exp Rheumatol 2018;36 Suppl 112:102-12.

22. Harada T, Ohashi T, Miyagishi R, Fukuda H, Yoshida K, Tagawa Y, Matsuda H. Optic neuropathy and acute transverse myelopathy in primary Sjögren's syndrome. Jpn J Ophthalmol 1995;39:162-5.

23. Rapoport P, Merle H, Smadja D. Ischemic choroidopathy in primary Sjögren's syndrome. Acta Ophthalmol Scand 1998;76:627-8.

24. Li H, Liu Z, Gong Y, Jiang Z, Zhang Y, Dai Y, Zhang $\mathrm{Y}$, Wei S. Application of immunosuppressant facilitates the therapy of optic neuritis combined with Sjögren's syndrome. Chin Med J (Engl) 2014;127:3098-104.

25. Yang JM, Sung MS, Ji YS, Heo H, Park SW. Analysis of Clinical Factors Associated with Retinal Morphological Changes in Patients with Primary Sjögren's Syndrome. PLoS One 2016;11:e0157995.

26. Scardina GA, Ruggieri A, Messina P. Periodontal disease and sjogren syndrome: a possible correlation? Angiology 2010;61:289-93.

27. Szabo N, Csiki Z, Szanto A, Danko K, Szodoray P, Zeher M. Functional and morphological evaluation of hand microcirculation with nailfold capillaroscopy and laser Doppler imaging in Raynaud's and Sjögren's syndrome and poly/dermatomyositis. Scand J Rheumatol 2008;37:23-9.

28. Alexander EL, Arnett FC, Provost TT, Stevens MB. Sjögren's syndrome: association of anti-Ro(SS-A) antibodies with vasculitis, hematologic abnormalities, and serologic hyperreactivity. Ann Intern Med 1983;98:155-9.

29. Sisto M, Lisi S, Ingravallo G, Lofrumento DD, D'Amore M, Ribatti D. Neovascularization is prominent in the chronic inflammatory lesions of Sjögren's syndrome. Int J Exp Pathol 2014;95:131-7. 
30. Sisto M, Lisi S, Lofrumento DD, D'Amore M, Frassanito MA, Ribatti D. Sjögren's syndrome pathological neovascularization is regulated by VEGF-A-stimulated TACE-dependent crosstalk between VEGFR2 and NF$\kappa$ B. Genes Immun 2012;13:411-20.

31. Sisto M, Lisi S, Lofrumento DD, D'Amore M, Ribatti D. Neuropilin-1 is upregulated in Sjögren's syndrome and contributes to pathological neovascularization. Histochem Cell Biol 2012;137:669-77.

32. Ozcaliskan S, Artunay O, Balci S, Perente I, Yenerel NM. Quantitative analysis of inner retinal structural and microvascular alterations in intermediate age-related macular degeneration: A swept-source OCT angiography study. Photodiagnosis Photodyn Ther 2020;32:102030.

33. Shin YI, Nam KY, Lee SE, Lim HB, Lee MW, Jo YJ, Kim JY. Changes in Peripapillary Microvasculature and Retinal Thickness in the Fellow Eyes of Patients With Unilateral Retinal Vein Occlusion: An OCTA Study. Invest Ophthalmol Vis Sci 2019;60:823-9.

34. Kim K, Kim ES, Kim DG, Yu SY. Progressive retinal neurodegeneration and microvascular change in diabetic retinopathy: longitudinal study using OCT angiography. Acta Diabetol 2019;56:1275-82.

35. Garcia-Martin E, Cipres M, Melchor I, GilArribas L, Vilades E, Polo V, Rodrigo MJ, Satue M.

Cite this article as: Yang QC, Yao F, Li QY, Chen MJ, Zhang LJ, Shu HY, Liang RB, Pan YC, Ge QM, Shao Y. Ocular microvascular alteration in Sjögren syndrome. Quant Imaging Med Surg 2022;12(2):1324-1335. doi: 10.21037/qims-21-234
Neurodegeneration in Patients with Type 2 Diabetes Mellitus without Diabetic Retinopathy. J Ophthalmol 2019;2019:1825819.

36. Spaide RF. Microvascular flow abnormalities associated with retinal vasculitis: a potential of mechanism of retinal injury. Retina 2017;37:1034-42.

37. Aspiotis M, Tsanou E, Gorezis S, Ioachim E, Skyrlas A, Stefaniotou M, Malamou-Mitsi V. Angiogenesis in pterygium: study of microvessel density, vascular endothelial growth factor, and thrombospondin-1. Eye (Lond) 2007;21:1095-101.

38. Cursiefen C, Maruyama K, Jackson DG, Streilein JW, Kruse FE. Time course of angiogenesis and lymphangiogenesis after brief corneal inflammation. Cornea 2006;25:443-7.

39. Kermorvant-Duchemin E, Sennlaub F, Sirinyan M, Brault S, Andelfinger G, Kooli A, Germain S, Ong H, d'Orleans-Juste P, Gobeil F Jr, Zhu T, Boisvert C, Hardy P, Jain K, Falck JR, Balazy M, Chemtob S. Transarachidonic acids generated during nitrative stress induce a thrombospondin-1-dependent microvascular degeneration. Nat Med 2005;11:1339-45.

40. Mariette X, Criswell LA. Primary Sjögren's Syndrome. N Engl J Med 2018;378:931-9. 